\title{
Queratocisto odontogênico: uma abordagem cirúrgica
}

\author{
Odontogenic keratocyst: a surgical approach \\ Queratoquiste odontogénico: un enfoque quirúrgico \\ Francielly Thomas FIGUEIREDO ${ }^{1}$ \\ Alana Oswaldina Gavioli Meira DOS SANTOS ${ }^{2}$ \\ Júlio Cesar Leite DA SILVA ${ }^{3}$ \\ José Carlos Garcia DE MENDONÇA ${ }^{3}$ \\ Gustavo Silva PELISSARO ${ }^{4}$ \\ Albert Schiavetto DE SOUZA ${ }^{3}$ \\ Ellen Cristina GAETTI-JARDIM ${ }^{3}$ \\ ${ }^{l}$ Cirurgiã-Dentista. Faculdade de Odontologia da Universidade Federal de Mato Grosso do Sul (Faodo-UFMS) \\ ${ }^{2}$ Residente em Cirurgia e Traumatologia Bucomaxilofacial da Faculdade de Odontologia da Universidade Federal de Mato Grosso do Sul (Faodo-UFMS) \\ ${ }^{3}$ Docente. Universidade Federal de Mato Grosso do Sul (Faodo-UFMS) \\ ${ }^{4}$ Doutorando. Universidade Federal de Mato Grosso do Sul (Famed-UFMS)
}

\section{Resumo}

O queratocisto odontogênico é uma neoplasia benigna onde lesões únicas ou múltiplas são encontradas em ossos ou cavidades. O objetivo deste trabalho é relatar um caso clínico de uma lesão de queratocisto odontogênico, evidenciando corretos diagnóstico e terapêutica. O relato de caso trata-se de um paciente de 32 anos, sexo feminino, melanoderma, que deu entrada à faculdade de odontologia da Universidade Federal de Mato Grosso do Sul, no ano de 2017, apresentando lesão radiolúcida com halo esclerótico em região de ângulo de mandíbula do lado esquerdo, sem envolvimento de dentes e/ou raízes adjacentes. Foi realizada uma tomografia computadorizada, constando hipótese diagnóstica radiográfica da mencionada patologia. O plano de tratamento adotado preconizou uma intervenção conservadora de descompressão e acompanhamento pós-operatório. A paciente se encontra em pós-operatório de 19 meses cirúrgico sem queixas álgicas e/ou funcionais.

Descritores: Cistos Ósseos; Descompressão; Terapêutica.

\section{Abstract}

Odontogenic keratocyst is a benign neoplasm where single or multiple lesions are found in bones or cavities. The objective of this study is to report a clinical case of an odontogenic keratocyst lesion, evidencing a correct diagnosis and therapeutics. The case report is a 32-year-old female, melanoderma, who was admitted to the dentistry faculty of the Federal University of Mato Grosso do Sul, in the year 2017, presenting a radiolucent lesion with halo sclerotic in an angle region of the left side of the jaw, without the involvement of adjacent teeth and / or roots. A CT scan was performed, with a radiographic diagnostic hypothesis of the aforementioned pathology. The adopted treatment plan advocated a conservative intervention of decompression and postoperative follow-up. The patient is in a year of the surgical procedure without pain and / or functional complaints.

Descriptors: Bone Cysts; Decompression; Therapy.

\section{Resumen}

El queratoquiste odontogénico es una neoplasia benigna en la que se encuentran lesiones únicas o múltiples en huesos o cavidades. El objetivo de este trabajo es informar un caso clínico de una lesión de queratoquiste odontogénico, mostrando un diagnóstico y tratamiento correctos. El informe del caso es una paciente de 32 años, melanoderma, que ingresó en la Facultad de Odontología de la Universidad Federal de Mato Grosso do Sul, en 2017, presentando una lesión radiotransparente con halo esclerótico en la región angulada. Mandíbula izquierda, sin afectación de dientes y / o raíces adyacentes. Se realizó una tomografía computarizada, con una hipótesis diagnóstica radiográfica de la patología mencionada. El plan de tratamiento adoptado recomendó una intervención conservadora de descompresión y seguimiento postoperatorio. El paciente se encuentra en el postoperatorio de 19 meses sin dolor y/o molestias funcionales.

Descriptores: Quistes Óseos; Descompresión; Terapéutica.

\section{INTRODUÇÃO}

$\mathrm{O}$ queratocisto odontogênico (QO) é uma neoplasia benigna de origem odontogênica de características peculiares quando comparadas aos demais tumores de cabeça e pescoço. Clinicamente é constituído por uma cavidade patológica revestida por epitélio e preenchida por substância fluida ou caseosa, conhecida pela comunidade científica como "amarelo citrino". Acredita-se que QO surja a partir do epitélio odontogênico, proveniente de remanescentes celulares da lâmina dentária ${ }^{2}$ demonstrando diferenciação nos mecanismos de crescimento e condição biológica.

Apesar do crescimento agressivo e alto índice de recorrência do QO após o tratamento, a Organização Mundial da Saúde atualizou a lista de cistos e tumores odontogênicos em 2017, reclassificando a nomenclatura, anteriormente referida como Tumor Odontogênico Queratocístico para Queratocisto Odontogênico ${ }^{3}$. Tais atualizações buscam padronizar o diagnóstico de patologias de cabeça e pescoço, visto que, as discussões atuais, relacionadas à classificação do queratocisto não foram conclusivas para tumor pela quantidade de evidências insuficientes para mantê-la como tal ${ }^{4}$.

O QO representa um tipo específico de cisto odontogênico não inflamatório, com crescimento agressivo e recidiva elevada, no entanto, sem apresentar manifestações sintomatológicas ${ }^{5}$. Essas recidivas podem ocorrer pela presença de cistos satélites unidos à lesão, bem como por sua organização delgada e friável dificultando a remoção cirúrgica por inteiro ${ }^{6}$. A sintomatologia, quando presente, pode estar relacionada ao tamanho da lesão, como os de dimensões maiores, apresentando edema, drenagem de material caseoso e dor associada, com uma tendência infiltrativa nos espaços medulares, crescendo de forma lenta, gradual e em sentido ântero-posterior, de modo que a expansão local seja observada tardiamente ${ }^{7}$.

Acomete $21,5 \%$ em relação aos cistos odontogênicos ${ }^{8}$ sendo na segunda e terceira década de vida, incluindo predileção para o sexo masculino 
em $58,4 \%$ dos $\operatorname{casos}^{9,10}$. Pode desenvolver em maxila e mandíbula, mas esta última costuma ser um local mais frequente, sobretudo nas regiões posterior, ângulo e ramo ascendente, não sendo, porém, características patognomônicas ${ }^{10,11}$.

Nos exames imaginológicos, apresentam-se como lesões únicas ( $80 \%$ dos casos), ou múltiplas $(16 \%)$, de tamanho variável, com halo esclerótico bem definido, podendo envolver um dente não erupcionado em cerca de $35 \%$ dos $\operatorname{casos}^{12}$. O diagnóstico diferencial segundo Meara et al. ${ }^{13} \mathrm{e}$ Scholl et al. ${ }^{14}$, é o cisto dentígero, ameloblastoma, cisto odontogênico calcificante, tumor odontogênico adenomatóide e fibroma ameloblástico, assim como cisto residual, cisto periodontal lateral, cisto ósseo traumático e cisto ósseo aneurismático ${ }^{5}$. Quando ocorrem lesões multiloculadas, as mesmas podem indicar associação à Síndrome do Carcinoma Nevoide de Células Basais. ${ }^{14}$

O diagnóstico definitivo do queratocisto odontogênico é dado pela análise histopatológica o que auxilia sobremaneira a escolha da terapêutica. Os cortes histológicos evidenciam um epitélio paraqueratinizado, apresentando de cinco a oito camadas de células epiteliais cuboidais ou colunares compondo a camada basal, dispensadas em paliçada e um núcleo hipercromático. Ocasionalmente podem ser observados cistos satélites na cápsula fibrosa corrugada e quando há um processo inflamatório, essas características histológicas podem ser alteradas $^{15,16}$.

Uma vez definido o diagnóstico de lesão cística, o tratamento é a excisão cirúrgica. A aspiração preventiva deve ser feita anteriormente à entrada na cavidade, sendo positiva e normalmente de cor amarelo citrino ${ }^{17}$. A descompressão ou a marsupialização são técnicas conservadoras, muito empregadas no tratamento das lesões císticas dos maxilares, que preservam estruturas nobres próximas à cavidade, e posteriormente, é realizada a enucleação $^{18}$. O uso adjunto da crioterapia, solução de Carnoy, curetagem e ostectomia, favorecem a diminuição da característica recidivante ${ }^{19,20}$.

\section{CASO CLÍNICO}

$\mathrm{O}$ relato de caso apresenta uma paciente de 32 anos, sexo feminino, melanoderma, que deu entrada à Faculdade de Odontologia - Faodo, da Universidade Federal de Mato Grosso do Sul UFMS, dezembro de 2017, encaminhada por cirurgião-dentista apresentando lesão radiopaca em ângulo e ramo ascendente de mandíbula esquerda compatível com neoformação óssea.

Após solicitação de novo exame de imagem, tomografia computadorizada, ao invés da referida neoformação óssea, ficou constatado lesão radiolúcida com halo esclerótico, $2,5 \mathrm{~cm}$ de tamanho, em região de ângulo e ramo ascendente de mandíbula do lado esquerdo, sem envolvimento de dentes e/ou raízes adjacentes (Figuras 1 e 2).

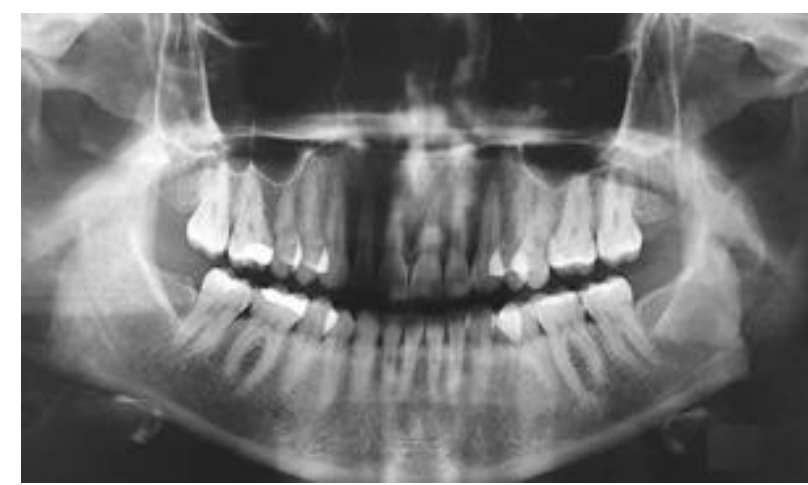

Figura 1: Radiografia panorâmica inicial evidenciando lesão óssea em região de ramo mandibular esquerdo.

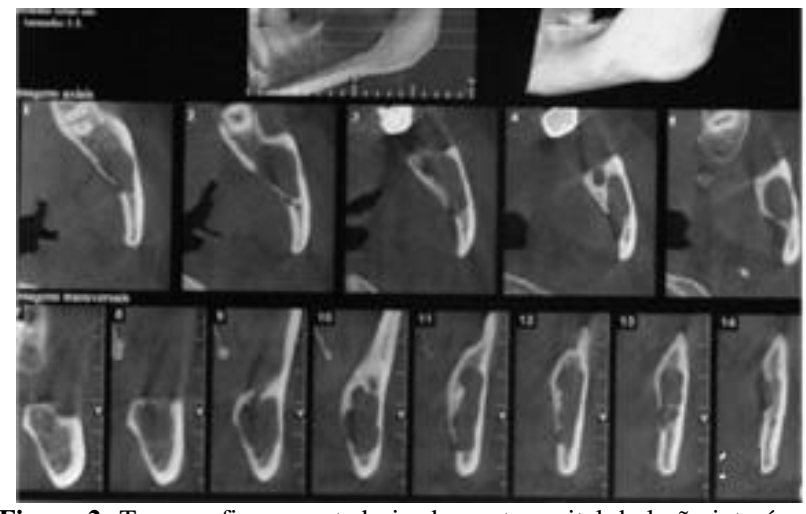

Figura 2: Tomografia computadorizada: corte sagital da lesão intraóssea em região de ramo mandibular esquerdo.

Clinicamente tanto extra como intrabucal nenhuma alteração da normalidade pode ser observada. A paciente relatava parestesia na região de ângulo de mandibular ipsilateral, ausência de queixas dolorosas ou quaisquer outros sintomas.

Em virtude das características imaginológicas e da sintomatologia associada, a ausência de características clinicas sugeriu o diagnóstico diferencial de queratocisto odontogênico. O plano de tratamento adotado compôs uma intervenção conservadora com descompressão da cavidade cística seguida de enucleação e acompanhamento pósoperatório.

Como medida pré-operatória, administração de dexametasona $8 \mathrm{mg}$ e amoxicilina $500 \mathrm{mg}$, ambos via oral, uma hora antes da cirurgia. Após, antissepsia extraoral com solução de clorexidina $2 \%$ em região peri-oral e labial e em seguida antissepsia intraoral com bochecho de digluconato de clorexidina a $0,12 \%$ durante 1 minuto. Fez-se anestesia tópica com Benzocaína 200mg/g aplicada em mucosa seca na área de punção de agulha para posterior anestesia por bloqueio regional dos nervos alveolar inferior, bucal e lingual todos do lado esquerdo.

Em seguida, uma punção aspirativa foi realizada com resultado de conteúdo semelhante a amarelo citrino. Posteriormente, incisão semilunar em região distal ao dente 37 seguida de descolamento mucoperiosteal e remoção do teto da cavidade cística 
com o auxilio de cinzel a pressão manual e envio da peça cirurgia para análise histopatológica. Após, foi colocado dispositivo de borracha no interior da cavidade cística e sutura do mesmo com nylon 5-0 a mucosa da região (Figuras 3, 4, 5 e 6). $\mathrm{O}$ mesmo permaneceu 15 dias em posição sendo a paciente orientada a realizar irrigação copiosa no interior do dispositivo ao menos três vezes ao dia com soro fisiológico $0,9 \%$.

Passados os 15 dias a borracha foi removida e a cavidade continuou sendo irrigada com soro fisiológico ate o fechamento completo da mucosa. Tanto pós-operatório imediato como os subsequentes a paciente não apresentou queixas, evidenciando o retorno a sensibilidade local já a partir do segundo mês após a cirurgia.

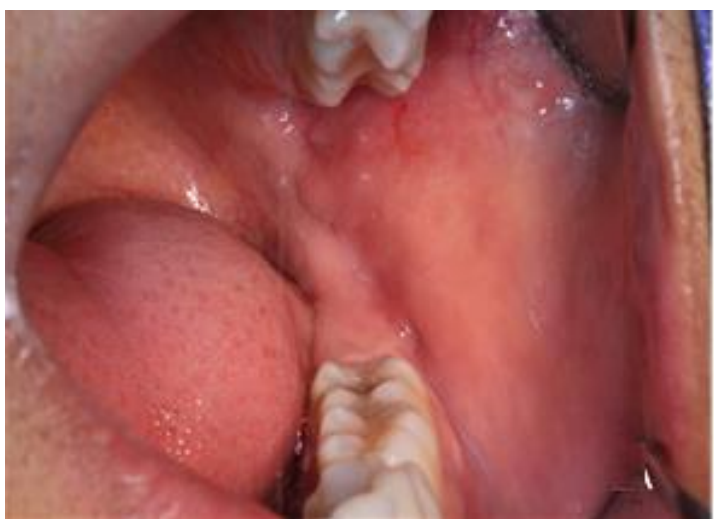

Figura 3: Aspecto clínico inicial da região de ramo mandibular esquerdo.

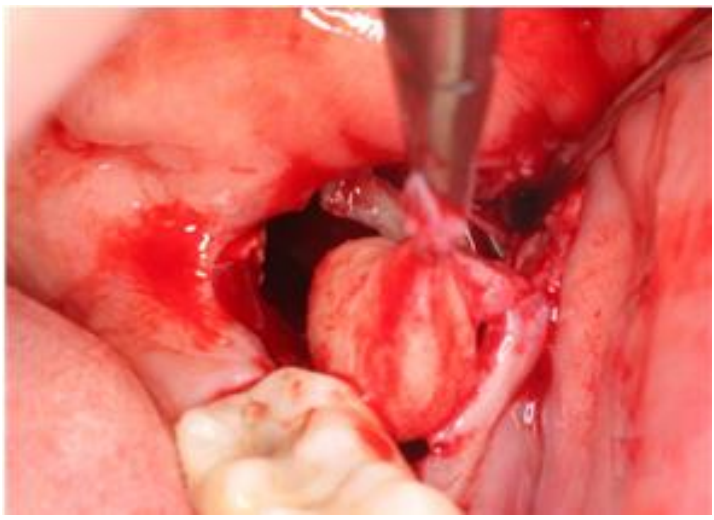

Figura 4: Exposição de cápsula cística em região de ramo mandibular esquerdo.

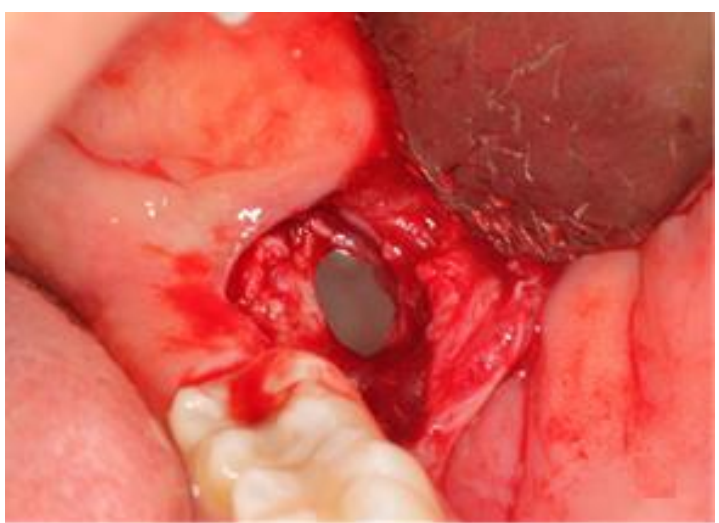

Figura 5: Remoção de cápsula cística de cavidade óssea.

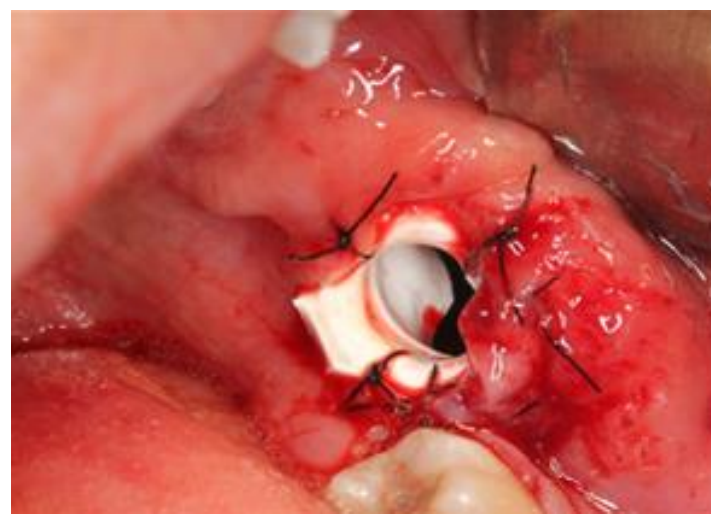

Figura 6: Dispositivo para descompressão de lesão cística suturado em mucosa bucal em região do ramo mandibular.

Os exames de imagem pós-intervenção foram realizados a cada 90 dias e apresentaram aspectos de normalidade (Figuras 7 e 8). A paciente se encontra no $19^{\circ}$ mês do procedimento e permanece sem quaisquer queixas e com retorno total da sensibilidade local bem como manutenção das funções mastigatórias além de neoformação óssea significativa.

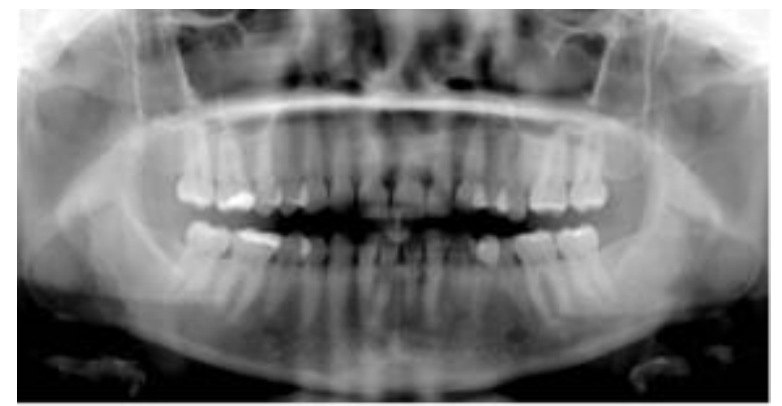

Figura 7: Radiografia panorâmica com dez meses de acompanhamento.

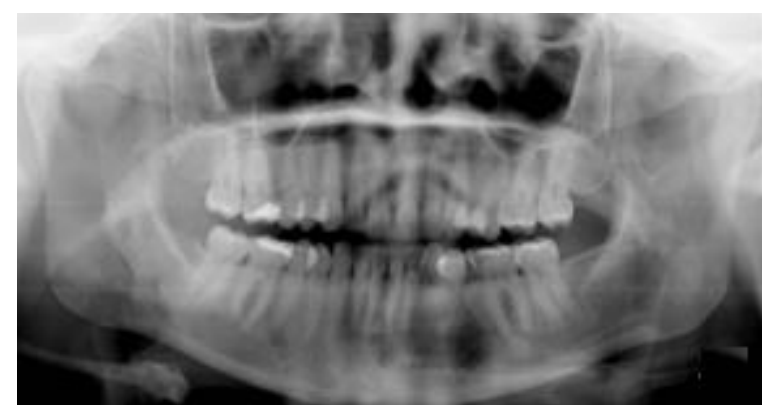

Figura 8: Radiografia panorâmica realizada dezenove meses após o procedimento cirúrgico.

\section{DISCUSSÃO}

Apesar da sua agressividade, diferentemente de outras patologias benignas maxilomandibulares, o queratocisto odontogênico possui como uma de suas principais características o não abaulamento das corticais ósseas $^{21,22}$. Fato este encontrado no caso apresentado, o que se mostra auxiliar na suposição do diagnóstico. Além disso, a presença de sintomatologia associada não é comum o que torna relevante tal relato de caso, no que diz respeito à presença de parestesia no trajeto do nervo alveolar inferior do mesmo lado do QO.

A faixa etária mais acometida pelo QO são a segunda e terceira décadas de $\operatorname{vida}^{22}$ o que foi 
corroborado por este caso, mas contra a característica de sexo uma vez que o mais acometido é o masculino e aqui foi associado ao feminino. A literatura expõe que a região anatômica com maior índice de acometimento pelo QO é a região posterior de mandíbula assim como em outros tumores dos maxilares o que também está de acordo com a apresentada neste caso ${ }^{23}$.

Associado a isto, outra característica fundamental do QO é o elevado índice de recidiva. Em um estudo de Moura et al. ${ }^{24}, 24 \%$ dos pacientes diagnosticados com QO apresentaram um quadro de recidiva. Desta forma, a escolha da descompressão ou marsupialização modificada como forma inicial de tratamento foi visando a necessidade de enucleação da lesão em etapa posterior, mesmo porque, a marsupialização em poucos casos configura como a terapêutica única empregada. Isso evidencia o bom resultado da técnica até o momento uma vez que a paciente passou apenas pela descompressão e o reparo ósseo local foi total e com retorno a sintomatologia local.

Shuster et al. ${ }^{1}$ e Wright et al. ${ }^{4}$ mencionam a utilização de técnicas coadjuvantes no tratamento do queratocisto quando associadas à exérese total da lesão como a solução de Carnoy, o uso da crioterapia, bem como a escarificação com brocas com ou sem irrigação de soro fisiológico, em virtude, novamente, da agressividade e do grau de recidiva ${ }^{25}$. Isto leva a crer o sucesso evidente da técnica empregada até o momento.

Em virtude de um trajeto insidioso e não produzir sintomatologia na grande maioria dos casos, precisar exatamente o tempo de evolução ou mesmo de surgimento do QO é tarefa quase impossível. Molon et al. $^{20}$ apontam forte associação do queratocisto odontogênico com a presença de terceiros molares. Uma vez que o fator etiológico não está bem estabelecido, o período de formação do germe do dente incluso pode coincidir ou mesmo ser fator causal do surgimento da lesão ${ }^{26}$. Sendo assim, fica a hipótese quanto à possibilidade de já estar presente quando da exodontia de tais dentes uma vez que a paciente relata ter removido há alguns anos. Fica aí a necessidade de realização de exames de imagem previamente aos procedimentos cirúrgicos.

A paciente encontra-se em 19 meses de acompanhamento pós-cirúrgico apresentando características de normalidade e ausência de sinais e sintomas de recidiva. Madras e Lapointe ${ }^{27}$ mencionam que a recidiva pode ainda ocorrer dentro de cinco anos da remoção de tal cisto, deste modo a paciente segue sob cuidados clínicos e imaginológicos periódicos.

\section{CONSIDERAÇÕES FINAIS}

Em virtude do que foi exposto, a terapêutica empregada se mostrou satisfatória com processo de reparo tecidual normal bem como retorno a sensibilidade configurando sucesso até o presente momento.

\section{REFERÊNCIAS}

1. Shuster A, Shlomi B, Reiser V, Kaplan I. Solid keratocystic odontogenic tumor report of a non agressive case. J Oral Maxillofac Surg. 2012;70(4):865-70.

2. Tsukamoto G, Sasaki A, Akiyama T, Ishikawa T, Kishimoto K, Nishiyama A, et al. A radiologic analysis of dentigerous cysts and odontogenic keratocysts associated with a mandibular third molar. Oral Surg Oral Med Oral Pathol Oral Radiol Endod. 2001;91(6):743-47.

3. Chan JKC, El-Naggar AK, Grandis JR, Takata T, Slootweg PJ. Who classification of head and neck tumours. World Health Organization 2017, 4th edition.

4. Wright JM, Vered M. Update from the 4th edition of the world health organization classification of head and neck tumours: odontogenic and maxillofacial bone tumors. Head Neck Pathology. 2017; 11(1): 68-77.

5. Gil JN, Rau Lh, Manfro R, Gasperini G, Dunker C, Chiarelli M. Ceratocisto odontogênico - caso clínico. Rev Port Estomatol Med Dent Cir Maxilofac. 2003;44(3):59-69.

6. Regezi JÁ, Sciubba JJ. Patologia bucal: correlações clinicopatológicas. 3. ed. Rio de Janeiro: Guanabara Koogan; 2000.

7. Larsen PE. Marsupialization for odontogenic keratocysts: Long-term follow-up analysis of the effects and changes in growth characteristics. 2002;94(5):543-53.

8. Gambhir A, Rani G. Conservative management of keratocystic odontogenic tumour with enucleation, excision of the overlying mucosa and electrocauterization: a case report. West Indian Med J. 2014;63(7):775-78.

9. Núñez-Urrutia S, Figueiredo R, Gay-Escoda C. Retrospective clinicopathological study of 418 odontogenic cysts. Med Oral Patol Oral Cir Bucal. 2010;15(5):e767-73.

10. González-Alva P, Tanaka A, Oku Y, Yoshizawa D, Itoh S, Sakashita $\mathrm{H}$, et al. Keratocystic odontogenic tumor: a retrospective study of 183 cases. J Oral Sci. 2008; 50(2):205-12.

11. Jones AV, Craig GT, Franklin CD. Range and demographics of odontogenic cysts diagnosed in a UK population over a 30-year period. J Oral Pathol Med. 2006;35(8):500-7.

12. Zhao YF, Wei JX, Wang SP. Treatment of odontogenic Keratocysts: a follow-up of 255 Chinese patients. Oral Surg Oral Med Oral Pathol Oral Radiol Endodo. 2002;94(2):151-56 .

13. Meara JG, Shah S, Li KK, Cunningham MJ. The odontogenic keratocyst: a 20-year 
clinicophatologic review. Laryngoscope. 1998;108(2):280-83

14. Kulkarni GH, Khaji SI, Metkari S, Kulkarni HS, Kulkarni R. Multiple keratocysts of the mandible in association with Gorlin-Goltz syndrome: A rare case report. Contemp Clin Dent. 2014;5(3):419-21.

15. Speight PM, Takata T. New tumour entities in the 4th edition of the world health organization classification of head and neck tumours: odontogenic and maxillofacial bone tumours. Virchows Arch. 2018;472(3):331-39.

16. Wright JM. The odontogenic keratocyst: orthokeratinized variant. Oral Surg. 1981;51(1):609-18.

17. Hupp JR, Ellis III E, Tucker MR. Cirurgia oral e maxillofacial contemporânea. 5. ed. Rio de Janeiro: Elsevier; 2009.

18. Dammer R, Niederdellmann H, Dammer P, Nuebler-Moritz M. Conservative or radical tretment of keratocysts: a retrospective review. $\mathrm{Br}$ J Oral Maxillofac Surg. 1997;35(1):46-8.

19. Browne RM. The pathogeneses of odontogenic cysts: a review. J Oral Pathol. 1975;4(1):31-46.

20. de Molon RS, Verzola MH, Pires LC, Mascarenhas VI, da Silva RB, Cirelli JA et al. Five years follow-up of a keratocyst odontogenic tumor treated by marsupialization and enucleation: A case report and literature review. Contemp Clin Dent. 2015;6(Suppl 1):S106-10.

21. Neville BW, Damm DD, Allen CM, Bouquot JE. Patologia oral e maxilofacial. 3.ed. Rio de Janeiro: Elsevier; 2009.

22. de Souza LB, Gordón-Núñez MAG, Nonaka CFW, de Medeiros MC, Torres TF, Emiliano GBG. Odontogenic cysts: Demographic profile in a Brazilian population over a 38-year period. Med Oral Patol Oral Cir Bucal. 2010;15(4):e583-90.

23. EL-Gehani R, Orafi M, Elarbi M, Subhashraj K: Benign tumours of orofacial region at Benghazi, Libya: a study of 405 cases. J Craniomaxillofac Surg 2009;37(7):370-75.

24. Moura BS, Cavalcante MA, Hespanhol W. Tumor odontogênico ceratocístico. Keratocystic odontogenic tumor. Rev Col Bras Cir. 2016; 43(6):466-71.

25. Kaczmarzyk T, Mojsa I, Stypulkowska J. A systematic review of the recurrence rate for keratocystic odontogenic tumour in relation to treatment modalities. Int J Oral Maxillofac Surg. 2012;41(6):756-67.

26. Lima GM, Nogueira RLM, Rabenhorst SHB. Considerações atuais sobre o comportamento biológico dos queratocistos odontogênicos. Rev Cir Traumatol Buco-Maxilo-fac. 2006;6(2):9-16.

27. Madras J, Lapointe H. Keratocystic odontogenic tumour: reclassification of the odontogenic keratocyst from cyst to tumour. J Canad Dent Assoc. 2008; 74(2):165-165h.

\section{CONFLITO DE INTERESSES}

Os autores declaram não haver conflitos de interesse.

\section{AUTOR PARA CORRESPONDENCIA}

\section{Alana Oswaldina Gavioli Meira dos Santos}

nagaviolli@gmail.com

Submetido em 11/09/2019

Aceito em 29/11/2019 